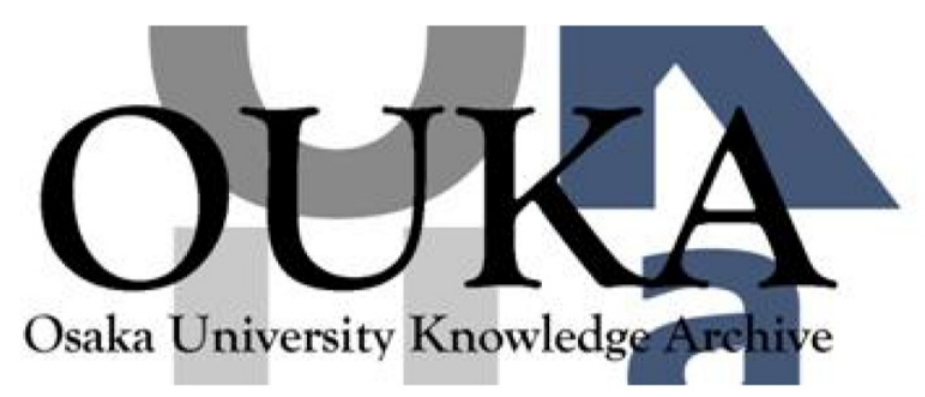

\begin{tabular}{|c|l|}
\hline Title & $\begin{array}{l}\text { Electrically color-tunable defect mode lasing } \\
\text { in one-dimensional photonic-band-gap system } \\
\text { containing liquid crystal }\end{array}$ \\
\hline Author(s) & $\begin{array}{l}\text { Ozaki, Ryotaro; Matsui, Tatsunosuke; Ozaki, } \\
\text { Masanori et al. }\end{array}$ \\
\hline Citation & Applied Physics Letters. 82(21) p. 3593-p. 3595 \\
\hline Issue Date & $2003-05-20$ \\
\hline oaire:version & VoR \\
\hline URL & https://hdl.handle.net/11094/75831 \\
\hline rights & \\
\hline Note & \\
\hline
\end{tabular}

Osaka University Knowledge Archive : OUKA

https://ir. Library. osaka-u. ac. jp/

Osaka University 


\section{Electrically color-tunable defect mode lasing in one-dimensional photonic-band-gap system containing liquid crystal}

Cite as: Appl. Phys. Lett. 82, 3593 (2003); https://doi.org/10.1063/1.1577829

Submitted: 02 January 2003. Accepted: 03 April 2003. Published Online: 20 May 2003

Ryotaro Ozaki, Tatsunosuke Matsui, Masanori Ozaki, and Katsumi Yoshino

\section{ARTICLES YOU MAY BE INTERESTED IN}

Electrically tunable lasing based on defect mode in one-dimensional photonic crystal with conducting polymer and liquid crystal defect layer

Applied Physics Letters 84, 1844 (2004); https://doi.org/10.1063/1.1686891

Temperature tuning of the stop band in transmission spectra of liquid-crystal infiltrated synthetic opal as tunable photonic crystal

Applied Physics Letters 75, 932 (1999); https://doi.org/10.1063/1.124558

Omnidirectional gap and defect mode of one-dimensional photonic crystals containing negative-index materials

Applied Physics Letters 83, 5386 (2003); https://doi.org/10.1063/1.1637452

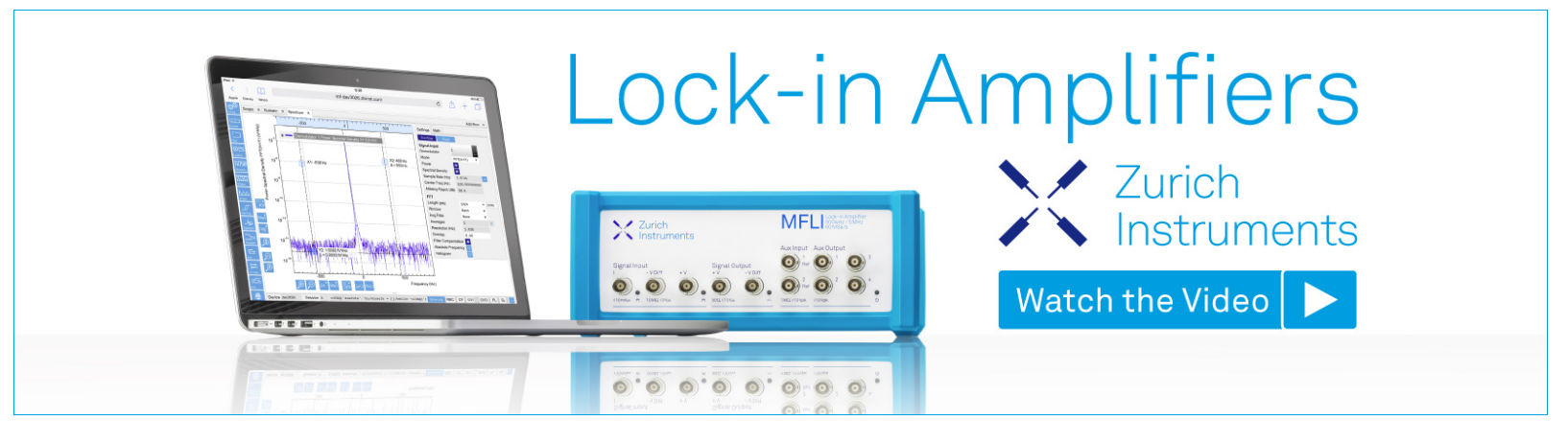




\title{
Electrically color-tunable defect mode lasing in one-dimensional photonic-band-gap system containing liquid crystal
}

\author{
Ryotaro Ozaki, Tatsunosuke Matsui, Masanori Ozaki, ${ }^{a}$ and Katsumi Yoshino \\ Department of Electric Engineering Graduate School of Engineering, Osaka University, 2-1 Yamada-Oka, \\ Suita, Osaka 565-0871, Japan
}

(Received 2 January 2003; accepted 3 April 2003)

\begin{abstract}
Electrical tuning of the wavelength of the defect-mode lasing in a one-dimensional periodic structure has been demonstrated using a dye-doped nematic liquid crystal as a defect layer in the periodic structure. Lasing wavelength is widely tuned upon applying an electric field, which is due to the refractive index change in the defect layer caused by the field-induced realignment of the liquid crystal molecules. (C) 2003 American Institute of Physics. [DOI: 10.1063/1.1577829]
\end{abstract}

Photonic crystal (PC) having a three-dimensional (3-D) ordered structure with a periodicity of optical wavelength has attracted considerable attention from both fundamental and practical points of view, because in such materials, a photonic band gap (PBG) exists in which the existence of a certain energy range of photons is forbidden. ${ }^{1,2}$ Physical concepts such as PBG have been theoretically predicted, and various applications of PCs have been proposed. In particular, the study of a defect mode in PBG is one of the most attractive subjects since photons are localized, and a lowthreshold laser based on the defect mode of the PCs should be expected. However, a complete 3-D PC with a periodicity equivalent to visible wavelength remains a technical challenge. ${ }^{3}$ Not only 3-D PCs but also one-dimensional (1-D) PCs are an attractive subject. Although the 1-D PC does not have a complete PBG, there are many applications using the extraordinary dispersion of the photon and the localized photonic state in a defect layer. So far, intensive studies on 1-D PC applications have been reported: air-bridge microcavities, ${ }^{4}$ the photonic band-edge laser,${ }^{5-7}$ the nonlinear optical diode, ${ }^{8}$ and the enhancement of optical nonlinearity. ${ }^{9,10}$

On the other hand, liquid crystals (LCs) have a large optical anisotropy and are sensitive to an external stress such as an electric field. Based on such optical anisotropy and field sensitivity, a tunable $\mathrm{PC}$ has been proposed in opal or inverse opal infiltrated with LC. ${ }^{11-16}$ Although opal and inverse opal are simple and inexpensive approachs to realize a 3-D PC using self-organization of colloidal particles, ${ }^{17,18}$ the introduction of a defect into the $3-\mathrm{D}$ periodic structure is a problem that must be resolved.

Recently, we have introduced a LC layer in a dielectric multilayer structure as a defect in a 1-D PC, ${ }^{19}$ in which the wavelength of defect modes were controlled upon applying electric field based on the change in optical length of the defect layer caused by the field-induced molecular reorientation of the LC. In this letter, we report a wavelength tunable laser based on an electrically controllable defect mode in a 1-D dielectric periodic structure containing a dye-doped LC as a defect layer. The lasing in a 1-D PC with a LC defect layer can be tuned over a wide range upon applying an electric field.

The 1-D PC with a LC defect is shown in Fig. 1. A dielectric multilayer consisting of an alternating stack of $\mathrm{SiO}_{2}$ and $\mathrm{TiO}_{2}$ layers deposited on an In-Sn-oxide (ITO)coated glass substrate is used as the 1-D PC. The center wavelength of the photonic band is adjusted to be $600 \mathrm{~nm}$ by setting the optical thickness of both $\mathrm{SiO}_{2}$ and $\mathrm{TiO}_{2}$ to be one-quarter of $600 \mathrm{~nm}$. The refractive indices of $\mathrm{SiO}_{2}$ and $\mathrm{TiO}_{2}$ are 1.46 and 2.35, respectively, and the thicknesses of $\mathrm{SiO}_{2}$ and $\mathrm{TiO}_{2}$ layers are 103 and $64 \mathrm{~nm}$, respectively. The number of $\mathrm{SiO}_{2}-\mathrm{TiO}_{2}$ pairs on each substrate is 5. The top surface of the dielectric multilayer is coated with a polyimide (Japan Synthetic Rubber, AL1254) and unidirectionally rubbed along the $y$-axis in Fig. 1.

In order to introduce the defect layer, a dye-doped nematic LC (Merck, E47) was sandwiched between substrates with a dielectric multilayer using $2-\mu \mathrm{m}$ spacers. The refractive index anisotropy $\Delta n$ of E47 is 0.209 at room temperature. In the absence of an electric field, the long molecular axis of the LC is aligned parallel to the substrates ( $y$-axis). As a laser dye dopant in the LC, [2[2-4-(dimethylamino) phenyl]ethenyl]-6-methyl-4H-pyran-4ylidene propanedinitrile, DCM (Exciton) is used. The concentration of the dye is $0.5 \mathrm{wt} \%$.

A rectangular wave voltage of $1 \mathrm{kHz}$ was applied between ITO layers to change the molecular alignment of the $\mathrm{LC}$ in the defect layer. In order to investigate the characteristics of the defect mode, the transmission spectrum of the linearly polarized light propagating along the $z$-axis was

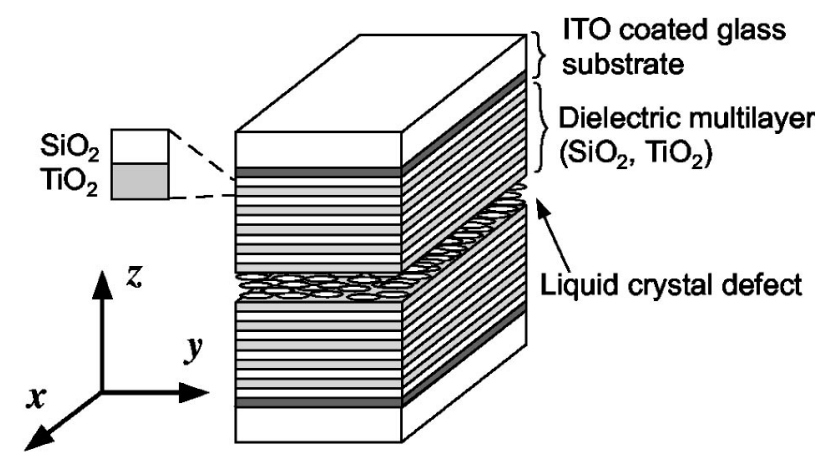

FIG. 1. One-dimensional PC with a LC defect.

${ }^{a)}$ Electronic mail: ozaki@ele.eng.osaka-u.ac.jp 


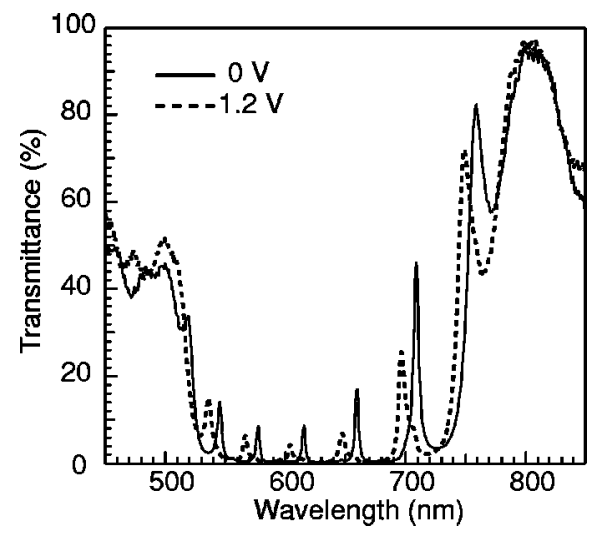

FIG. 2. Transmission spectra of 1-D PC with a LC defect as a function of applied voltage.

measured from the opposite side of the cell using a CCD multichannel spectrometer (Hamamatsu Photonics, PMA-11) having a spectral resolution of $3 \mathrm{~nm}$.

A second-harmonic light of a Q-switched Nd:YAG laser (Spectra Physics, Quanta-Ray INDI) is used for excitation, whose wavelength, pulse width, and pulse repetition frequency are $532 \mathrm{~nm}, 8 \mathrm{~ns}$, and $10 \mathrm{~Hz}$, respectively. The illumination area on the sample is about $0.2 \mathrm{~mm}^{2}$. The excitation laser beam irradiated the sample perpendicularly to the cell plate. The emission spectra from the 1-D PC with a dye-doped nematic LC are measured from the opposite side of the cell using the CCD multichannel spectrometer. In order to control the emission wavelength, the orientation of the LC molecules in the defect layer was changed upon applying a rectangular wave voltage of $1 \mathrm{kHz}$.

Figure 2 shows the transmission spectra for the incident light polarized along the $y$-axis, which corresponds to the rubbing direction and the initial orientation direction of the LC molecules in the defect layer, as a function of the applied voltage. The solid and broken lines correspond to spectra at 0 $\mathrm{V}$ and $1.2 \mathrm{~V}$, respectively. Regardless of the application of voltage, PBGs are observed in the spectral range from 520 to $780 \mathrm{~nm}$, and these bandwidths do not depend on the voltage. The slight decrease in the transmittance at shorter wavelengths is attributed to the absorption of DCM. The peaks of the defect modes shift to shorter wavelengths upon applying voltage. This peak shift originates from the decrease in the optical length of the defect layer caused by the field-induced reorientation of the LC molecules. ${ }^{19}$ Therefore, we confirmed that the wavelength of the defect mode in 1-D PC with the dye-doped LC layer can be controlled upon applying voltage.

Figure 3(a) shows photoluminescence spectrum of the DCM-doped LC without a 1-D PC. Figure 3(b) shows the emission spectra of a 1-D PC with the dye-doped LC as a function of the applied voltage. A sharp emission peak appears. Figure 4 shows the pump-energy dependence of the peak intensity at $0 \mathrm{~V}$. Above the threshold at a pump-pulse energy of about $3 \mu \mathrm{J}$, the emission intensity drastically increases. This indicates that there exists a lasing threshold above which a light amplification accrues. The full width at half-maximum (FWHM) of the lasing peak is about $3 \mathrm{~nm}$, which is limited by the spectral resolution of the CCD spectrometer used in this experiment. The FWHM of this emission peak is narrower than that of the transmission peak of



(a)

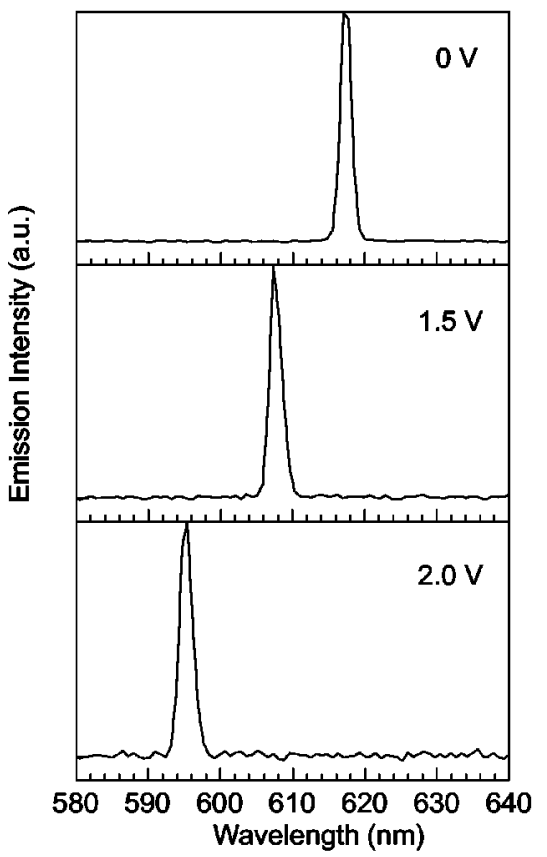

(b)

FIG. 3. (a) Photoluminescence spectrum of the dye-doped LC. (b) Emission spectra of 1-D PC with the dye-doped LC defect as a function of applied voltage.

the defect mode $(10 \mathrm{~nm})$ shown in Fig. 2. This indicates that the emission peak in the band is not the spontaneous emission of DCM passing through the narrow band window of a defect mode.

It should be noted that lasing peak shifts toward shorter wavelengths with increasing voltage in the same manner as the defect modes shift (see Fig. 2). The wavelength shift of the lasing peak is about $25 \mathrm{~nm}$, even upon applying low voltage. Figure 5 shows voltage dependence of the lasing peak wavelength. As is evident from Fig. 5, a threshold voltage exists for the peak shift, and the lasing wavelength shifts

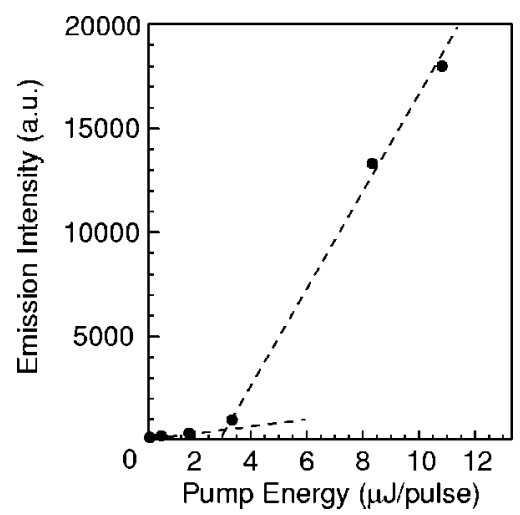

FIG. 4. Pump-energy dependence of the peak intensity. 




FIG. 5. Voltage dependence of the defect-mode lasing wavelength.

toward shorter wavelengths above the threshold of $1.1 \mathrm{~V}$. This is associated with the Fredericks transition of the LC in the defect layer. Assuming the dielectric constants of LC, $\mathrm{TiO}_{2}$, and $\mathrm{SiO}_{2}$ to be 4,100, and 4, respectively, the effective voltage applied to the LC layer is estimated to be $0.82 \mathrm{~V}$. The threshold voltage evaluated for the Fredericks transition as the voltage at $90 \%$ of the maximum transmission change in a conventional twisted nematic cell is $1.49 \mathrm{~V}$. However, this threshold is much higher than the true Fredericks threshold voltage above which the LC molecules start to move due the voltage application. In the 1-D PC with LC defect, the defect-mode wavelength is markedly sensitive to the refractive index change of the LC layer, and the threshold shown in Fig. 5 should correspond to the real Fredericks threshold.

In Fig. 5, the discontinuous change of the lasing wavelength is observed around $2.1 \mathrm{~V}$. This means that the defect mode at shorter wavelengths go out of the wavelength range of the high emission efficiency of DCM above $2.1 \mathrm{~V}$. On the other hand, the defect mode peak at the longer wavelengths appears in the lasing range above $2.3 \mathrm{~V}$.

In conclusion, we demonstrated the electrical tuning of the defect-mode lasing in a 1-D PBG by using a dye-doped nematic liquid crystal as a defect layer. Laser emission was observed upon the irradiation of pump laser beam above the threshold energy. Lasing wavelength was widely tuned with a low voltage.

This work is partially supported by a Grant-in-Aid for Scientific Research (14350165) from the Japan Ministry of Education, Culture, Sports, Science and Technology.

${ }^{1}$ E. Yablonovitch, Phys. Rev. Lett. 58, 2059 (1987).

${ }^{2}$ S. John, Phys. Rev. Lett. 58, 2486 (1987).

${ }^{3}$ S. Noda, K. Tomoda, N. Yamamoto, and A. Chutinan, Science (Washington, DC, U.S.) 289, 604 (2000).

${ }^{4}$ J. S. Foresi, P. R. Villeneuve, J. Ferrera, E. R. Thoen, G. Steinmeyer, S. Fan, J. D. Joannopoulos, L. C. Kimerling, H. I. Smith, and E. P. Ippen, Nature (London) 390, 143 (1997).

${ }^{5}$ J. P. Dowling, M. Scalora, M. J. Bloemer, and C. M. Bowden, J. Appl. Phys. 75, 1896 (1994).

${ }^{6}$ V. I. Kopp, B. Fan, H. K. M. Vithana, and A. Z. Genack, Opt. Lett. 23, 1707 (1998).

${ }^{7}$ M. Ozaki, M. Kasano, D. Ganzke, W. Haase, and K. Yoshino, Adv. Mater. (Weinheim, Ger.) 14, 306 (2002).

${ }^{8}$ M. D. Tocci, M. J. Bloemer, M. Scalora, J. P. Dowling, and C. M. Bowden, Appl. Phys. Lett. 66, 2324 (1995).

${ }^{9}$ T. Hattori, N. Tsurumachi, and H. Nakatsuka, J. Opt. Soc. Am. 14, 348 (1997).

${ }^{10}$ Y. Dumeige, P. Vidakovic, S. Sauvage, I. Sgnes, and J. A. Levenson, Appl. Phys. Lett. 78, 3021 (2001).

${ }^{11}$ K. Yoshino, S. Satoh, Y. Shimoda, Y. Kawagishi, K. Nakayama, and M. Ozaki, Jpn. J. Appl. Phys. 38, L961 (1999).

${ }^{12}$ K. Yoshino, Y. Shimoda, Y. Kawagishi, K. Nakayama, and M. Ozaki, Appl. Phys. Lett. 75, 932 (1999).

${ }^{13}$ K. Busch and S. John, Phys. Rev. Lett. 83, 967 (1999).

${ }^{14}$ Y. Shimoda, M. Ozaki, and K. Yoshino, Appl. Phys. Lett. 79, 3627 (2001).

${ }^{15}$ D. Kang, J. E. Maclennan, N. A. Clark, A. A. Zakhidov, and R. H. Baughman, Phys. Rev. Lett. 86, 4052 (2001).

${ }^{16}$ M. Ozaki, Y. Shimoda, M. Kasano, and K. Yoshino, Adv. Mater. (Weinheim, Ger.) 14, 514 (2002).

${ }^{17}$ K. Yoshino, K. Tada, M. Ozaki, A. A. Zakhidov, and R. H. Baughman, Jpn. J. Appl. Phys. 36, L714 (1997).

${ }^{18}$ Y. A. Vlasov, K. Luterova, I. Pelant, B. Honerlage, and V. N. Astratov, Appl. Phys. Lett. 71, 1616 (1997).

${ }^{19}$ R. Ozaki, T. Matsui, M. Ozaki, and K. Yoshino, Jpn. J. Appl. Phys. 41, L1482 (2002). 